\title{
SPATIO-TEMPORAL ANALYSIS OF DENGUE AND WEATHER VARIABLES IN YOGYAKARTA
}

\author{
Rinaldi Daswito ${ }^{1)}$, Lutfan Lazuardi'2), Hera Nirwati3) \\ 1) Department of Public Health, Faculty of Medical and Health Science, \\ Jambi University; ${ }^{2}$ Department of Public Health, Faculty of \\ Medicine, Gadjah Mada University; 3)Department of Microbiology, \\ Faculty of Medicine, Gadjah Mada University
}

\begin{abstract}
BACKGROUND: Dengue Hemorrhagic Fever (DHF) is one of the main public health issues in Indonesia, and is endemic in all provinces. The annual incidence of DHF has been fluctuated in Yogyakarta. This study aimed to examine the pattern of the relationship among weather variables on the incidence of dengue in the city of Yogyakarta for 5 years (20102014).

SUBJECT AND METHODS: This was an ecological study with spatiotemporal approach. The study population was the incidence of dengue for the period 2010-2014 in Yogyakarta city. The independent variables in this study were rainfall, temperature, relative humidity, and wind velocity. The dependent variable was the incidence of DHF. The data was analyzed using graphic/time-trend and spatial statistical analysis. Rainfall in theory increases mosquito breeding place. In this study, however, rainfall was not included in fitting the regression analysis model.

RESULTS: There was a relationship among weather variable with dengue incidence, both in graphs/time-trend and spatial statistic analyses. In the graph/time-trend and spatial analysis, there was a relationship between dengue outbreaks in 2010 and 2013 in rainfall fluctuations and temperature. Two regressions models were used, i.e. Poisson Regression and Negative Binomial Regression. The smallest Akaike's Information Criterion (AIC) and Bayesian Information Criterion (BIC) values were used to indicate model fit with respect to the contribution of weather variables on dengue incidence. The incidence of dengue at a lag of one month, temperature of the same month, temperature at the lag of two months, relative humidity of the same month, relative humidity at the lag of three months, and wind velocity at the lag of two months, were predictors for dengue incidence.

CONCLUSIONS: The pattern of dengue incidence following climate fluctuation, rainfall lag of two months, temperature lag of three months, humidity in lag of one month, had positive association with increased incidence of dengue. Wind velocity was inversely associated with dengue incidence of the same month. The policy implication of the study is that the Health Office of Yogyakarta city should consider climatological data in surveillance and planning dengue prevention program.
\end{abstract}

Keywords: dengue hemorrhagic fever, vector-borne disease, climate, spatio-temporal analysis 